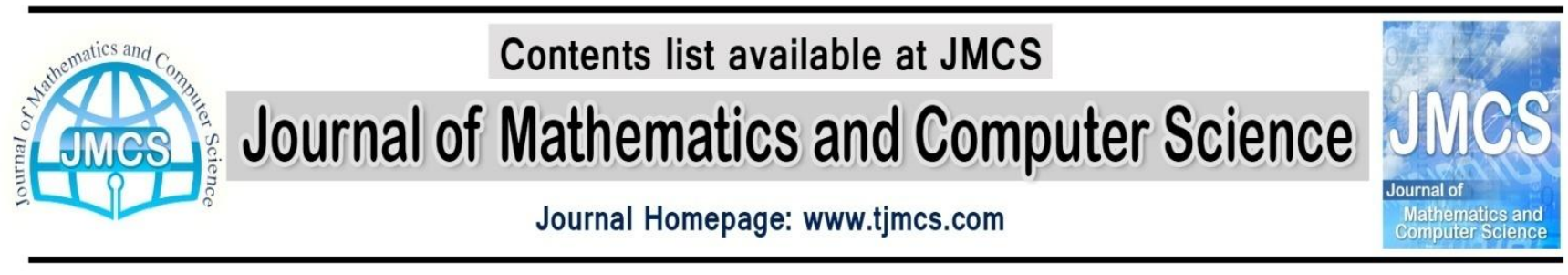

\title{
Design an Optimal T-S Fuzzy PI Controller for a Non-Inverting Buck-Boost Converter
}

\author{
Omid Naghash Almasi ${ }^{1^{*}}$, Vahid Fereshtehpoor ${ }^{2}$, Abolfazl Zargari ${ }^{3}$, Ehsan Banihashemi ${ }^{4}$ \\ ${ }^{1,2}$ Young Researchers and Elite Club, Mashhad Branch, Islamic Azad University, Mashhad, Iran \\ ${ }^{3}$ Department of Electrical Engineering, Najafabad Branch, Islamic Azad University, Isfahan, Iran \\ ${ }^{4}$ Department of Computer Science, Ferdows Branch, Islamic Azad University, Ferdows, Iran

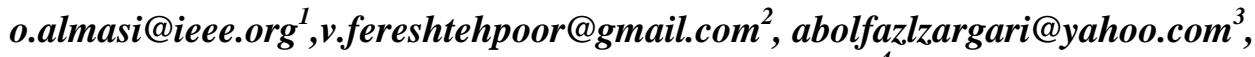 \\ banihashemi.ehsan@gmail.com ${ }^{4}$
}

Article history:

Received: April 2014

Accepted: May 2014

Available online : June 2014

\begin{abstract}
In this paper, first, the different operation modes of a non-inverting buck-boost converter are examined, and then an optimal T-S fuzzy PI controller is proposed to control the converter under variable reference voltages. The controller is designed based on input-output pairs of the classic PI controller to employ both conscious and subconscious knowledge. For this aim, the initial fuzzy system generated by subtractive clustering method and then the Recursive Least Mean Square (RLS) is used to adjust the coefficients of consequent part of fuzzy rules. Simulation and experimental results show the superior control performance of the fuzzy PI controller over the classic PI controller.
\end{abstract}

Keywords: Fuzzy PI controller; Non-inverting Buck-boost Converter; TSK fuzzy systems.

\section{Introduction}

In many industrial applications, it is necessary to convert an unregulated DC input voltage into a regulated DC output voltage. DC-DC converters are used in personal computers, laptops, Personal Digital Assistants (PDAs), office appliances, aircrafts, satellite communication equipment and DC motor starting circuits $[1,2]$. Therefore, there has been a growing interest in the development of efficient control strategies to improve dynamic behavior of DC-DC converters.

There are different types of switching DC-DC converters, among which the boost regulator is used in applications where the output voltage should be higher than the input and the output polarity is the same as the input voltage [3].

Non-inverting buck-boost converter is combination of a buck and a boost converter with two switches, an inductor, and a capacitor. The extra switch and a diode solve the problems of the aforementioned converters. In addition, non-inverting buck-boost converter is able to be used in 
three different separate operation modes, i.e. buck (only S1 is switched within one switching period), boost (only S2 is switched within one switching period), and buck-boost (both S1 and S2 are switched) [4-6].

The most common method to model power electronic converters is the state-space averaging approach [7, 8]. While this model is obtained for the boost converter, a non-linear equation is appeared, which first requires linearization around the operating point to obtain the small signal transfer function of the converter system. In other words, the desired transfer function is only valid in vicinity of the operating point and for a small range of variations in system parameters. This would also be true for the controller designed based on the transfer function. As aforementioned, if a wide range of the output voltage is desired and because of the non-minimum phase characteristic of the transfer function of the boost converter, one will face severe restrictions in selecting a wide bandwidth for the controller.

In recent years, simple structures and the ability of Takagi-Sugeno and Kang (TSK) fuzzy systems to model the expert knowledge with the aim of controlling and describing the nonlinear systems have been attracted the attention of the researchers [9].

The knowledge base of a fuzzy system can be designed based on an expert knowledge or inputoutput pairs [10]. In the design of the TSK system, experts face two major issues: 1) defining the assumptions of the antecedent part in the fuzzy rules 2) determining appropriate coefficients for the consequent part of the fuzzy rules.

Generally, in complex systems resolving this problem may take a long time. Moreover, the knowledge base designed based on the expert knowledge has the lower accuracy in comparison with knowledge base designed based on the input-output pairs [11].

The reason of loss in accuracy is that the experts are only able to formulate their conscious human knowledge in form of a set of IF-THEN fuzzy rules and unable to formulate their subconscious knowledge. Therefore, several methods have been proposed for formulating both conscious and subconscious knowledge [12-14].

In these methods, the expert is considered as a black box, and the input-output pairs are collected. The conscious and subconscious knowledge are hidden in a set of input-output pairs. Thus, by developing the fuzzy system based on the input-output pairs, both types of knowledge can be utilized.

To extract fuzzy rules from the input-output pairs, various methods such as Kohnen's neural networks [12] and fuzzy clustering [13] have been used. In these methods, first the number of clusters needs to be defined (extracting the number of predetermined rules). In this study, the subtractive clustering is used because, unlike other methods, it does not require to determine the number of clusters, i.e. the number of rules is obtained automatically [14]. After extracting the fuzzy rules and determining the antecedent assumptions by differential clustering method, the Recursive Least Square (RLS) method based on the least square means error between the optimal output and the output of TSK fuzzy system is used to regulate the coefficients of the consequent part.

In this paper, an optimal T-S fuzzy PI controller is suggested to control a non-inverting buck-boost converter in different reference voltages. The controller is designed by using the input-output pairs obtained from the classic PI controller to employ both conscious and subconscious knowledge. For this purpose, the initial fuzzy system generated by subtractive clustering method and then the Recursive Least Mean Square (RLS) is used to adjust the coefficients of consequent part of fuzzy rules. 
Table 1- Switching strategies of non-inverting buck-boost converter

\begin{tabular}{ccc}
\hline Mode & $\mathbf{S}_{\mathbf{1}}$ & $\mathrm{S}_{\mathbf{2}}$ \\
\hline Buck & Switching & Off \\
Boost & On & Switching \\
Buck-Boost & Switching & Switching \\
\hline
\end{tabular}

The rest of this paper is organized as follows. In section II, the linearized averaged state-space model of the non-inverting converter is obtained and analyzed. Section III describes the formulation and the construction method of TSK fuzzy system. The fuzzy PI controller is presented in section IV. Some simulations and experimental results are presented in support of the proposed controller in section V. Finally, conclusions are drawn in section VI.

\section{Linearized averaged state space model and its analysis}

In this section, first the linearized averaged state space model of non-inverting buck-boost converter is presented in subsection 2.1, and then the frequency domain analysis of the model is presented in subsection 2.2 .

\subsection{Linearized averaged state space model}

In order to obtain and analyze the state space model, the switching table and the various operating modes of this converter should be considered. Table1 shows the switching strategies of this converter in different modes. Generally, control system must operate in such a way that buck functional state occurs when the output voltage of the converter is lower than its input voltage. In this case, the switch S2 will be turned OFF during the switching interval and only the switch S1 will be switched ON and OFF. If the output voltage is greater than the input voltage, the converter must change into boost mode, in which the S1 switch remains constantly turned ON during the switching interval and switch S2 is switched. If both S1 and S2 are switched simultaneously with the identical duty cycle, the non-inverting buck-boost converter will be similar to the conventional buck-boost converter in terms of performance, though here the polarity of the output voltage is positive and the stress on the switches and diodes is remarkably reduced.

The important point is that in buck-boost mode, since both switches are turned ON and OFF, the switching losses is higher than other modes, which can reduce the convertor's efficiency. Thus, in this paper, to avoid this problem the converter is controlled in a way that buck-boost functional state is not used at all.

In other words, depending on the amplitude of input and output voltage, the converter can only be in buck or boost modes. A small signal transfer function is achieved by obtaining the averaging statespace model and linearizing it around its operating point.

The transfer function finds the relation between the capacitor's voltage, control input, and chaos input in both buck (1) and boost modes (2) as follows [5]:

$$
\hat{V_{c}}=\frac{\frac{V_{i n}}{L C}}{s^{2}+\frac{s}{R_{o} C}+\frac{1}{L C}} \hat{d}+\frac{\frac{D_{1}}{L C}}{s^{2}+\frac{s}{R_{o} C}+\frac{1}{L C}} \hat{V}_{\text {in }}
$$




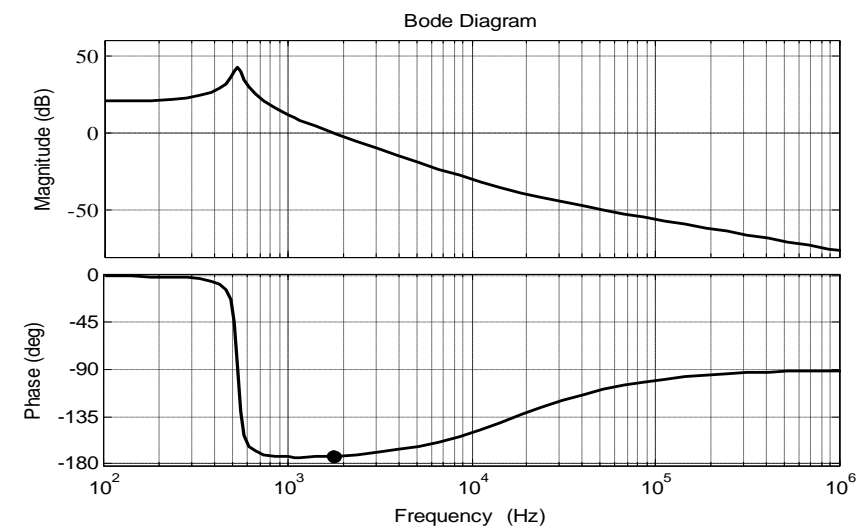

(a)

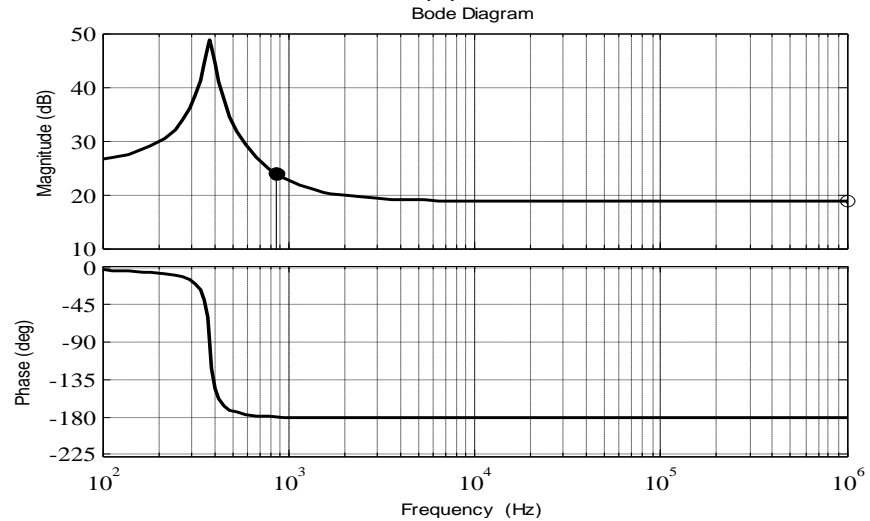

(b)

Figure 1- Frequency response diagram, (a) buck mode, (b) boost mode

$$
\hat{V}_{c}=\frac{\frac{i_{L}}{C}\left(\frac{V_{i n}}{L i_{L}}-s\right)}{s^{2}+\frac{s}{R_{o} C}+\frac{\left(1-D_{2}\right)^{2}}{L C}} \hat{d}+\frac{\frac{\left(1-D_{2}\right)}{L C}}{s^{2}+\frac{s}{R_{o} C}+\frac{\left(1-D_{2}\right)^{2}}{L C}} \hat{V}_{i n}
$$

where $R_{0}, L$ and $C$ are load resistance, inductor's inductance and capacitor capacitance, respectively. $D_{1}$ and $D_{2}$ are duty cycles of the switches $S_{1}$ and $S_{2} ; i_{1}$ is the inductor current, and $v_{i n}$ is the steady state input voltage. Moreover, the superscript $\wedge$ represents small AC variations around the steadystate operating point.

Among the parasitic elements, only the effect of the equivalent series resistance of the capacitor $\left(R_{c}\right)$ which creates a zero in the transfer function of the system should be incorporated in the transfer function. The other parasitic elements due to their small values will not noticeably affect the parameters of the transfer function. Therefore, the other parasitic elements can be omitted from the small-signal analysis of the circuit. Equation (3) presents the relationship between the output voltage and capacitor voltage

$$
\hat{V}_{o}=\left(1+R_{C} C s\right) \hat{V}_{C}
$$

The small-signal transfer function of the output voltage in order to control input for buck mode is expressed as follows [5]: 


$$
\hat{v}_{o}=\frac{\frac{V_{i n}}{L C}\left(1+R_{c} C s\right)}{s^{2}+\frac{s}{R_{o} C}+\frac{1}{L C}} \hat{d}
$$

and for the boost operation, the transfer function is as follows [5]:

$$
\hat{v_{o}}=\frac{\frac{i_{L}}{C}\left(\frac{V_{\text {in }}}{L i_{L}}-s\right)\left(1+R_{C} C s\right)}{s^{2}+\frac{s}{R_{o} C}+\frac{\left(1-D_{2}\right)^{2}}{L C}} \hat{d}
$$

\subsection{Linearized averaged state space model}

The frequency response analysis along with Bode diagrams was used to analyze the different operating points of the converter. Figure 1 shows the Bode diagram for this converter in (a) buck mode and (b) boost mode. As it can be seen, the stability margins of the system is quite positive in buck mode, although the converter model has negative phase and gain margins in boost mode which makes it completely unstable. The transfer function evaluation of the system shows an unstable zero whose position depends on the inductor value, the inductor current, and the input voltage level. This unstable zero imposes some restrictions on the choice of a wide bandwidth for the controller [15-17].

To sum up, the negative phase created by the right half plane zero prevents the designer from selecting a high cut-off frequency. This restriction choice slows the dynamics of the system.

\section{TSK Fuzzy Systems}

TSK fuzzy systems are universal approximations, which mean they are able to approximate any function or system to any desired accuracy $[11,18]$. In TSK fuzzy system, the antecedent part (IF) of the IF-THEN rules of the fuzzy system are identical to the typical fuzzy systems (Mamdani), but the consequent part (THEN) is a linear combination of the input variables [19]. Thus, this type of fuzzy system does not have defuzzification part. In fact, TSK fuzzy system can be described as the mean weight of values obtained from the "THEN" part of the rules. Every fuzzy rule in the TSK fuzzy system is shown as follows:

$$
\mathrm{R}_{\mathrm{i}}: \mathrm{IF} x \text { is } A_{i} \text { THEN } y_{i}=a_{i}^{T} x+b_{i},(i=1,2, \ldots, M)
$$

where $x \in R^{n}$ and $y \in R$ are input and output variables of $i$-th fuzzy rule, respectively. $M$ represents the number of rules and ${ }_{i}$ is the fuzzy variable of "IF" part in $[0,1] \in R^{n}$ interval. The antecedent part in the fuzzy system is summarized as follows:

$$
A_{i}(x)=\prod_{j=1}^{n} \mu_{i j}\left(x_{j}\right)
$$

For the $k$-th input $x_{k}$, the total output $y(k)$ of the system is computed by aggregating the individual rules contributions 


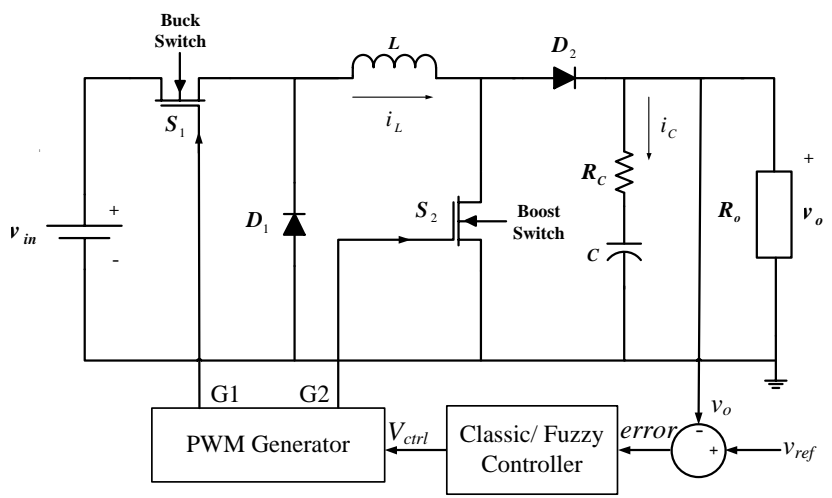

Figure 2- The proposed control scheme for controlling non-inverting buck-boost converter

$$
y(k)=\sum_{i=1}^{M} \mu_{k i} y_{i}(k)
$$

where ${ }^{\mu} k i$ is the normalized degree of fulfillment of the antecedent clause of rule $R_{i}$.

$$
\mu_{k i}=\frac{A_{i}\left(x_{k}\right)}{\sum_{i^{\prime}=1}^{M} A_{i^{\prime}} x_{k}}
$$

Knowledge rule base is the most important part of TSK fuzzy system. In designing knowledge rule base using classic methods, the fuzzy rules should be determined by an expert who has deep knowledge about the system operation. This expert should also appropriately choose the type of the membership functions for modeling the inputs of the fuzzy system. Dispensable choosing a large number of rules will be complicated the fuzzy systemandalso the obtained model occupies large amount of memory, which can reduce the process speed. In contrast, choosing a small number of rules generates a weak fuzzy system that may not fulfill the proposed objective $[10,11]$.

As discussed earlier, in order to incorporate both conscious and subconscious knowledge in developing the fuzzy system, the differential clustering method was used to create the initial fuzzy system $[14,20]$.

The initial model is not optimal because the antecedent part of the rules was achieved only based on partitioning the input-output space. Thus, the model is not able to portray the relation between input and output adequately.

Several methods such as gradient descent and least mean squares by taking an error measure, such as Mean Square Error (MSE), between the output of TSK fuzzy system and the optimal output adjust the parameters of the initial fuzzy system. In this study, the RLS method due to its higher speed and lower memory occupation compared to other approaches is used for optimizing initial TSK fuzzy systems $[10,11]$.

For a thorough study of the clustering methods, see references $[14,20]$. MSE is defined as follows: 


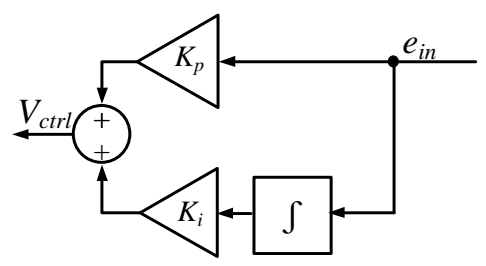

Figure 3- Schematic model of the classic PI controller

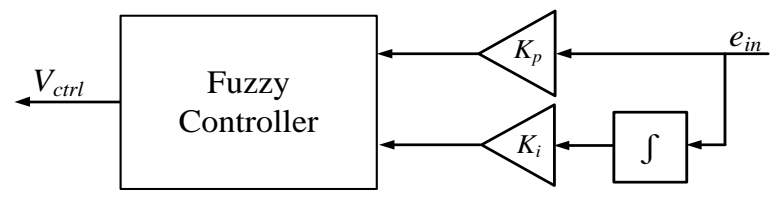

Figure 4-Schematic model of fuzzy PI controller

$$
M S E=\frac{1}{K} \sum\left(y-y_{d}\right)^{2}
$$

wherey is the output of TSK fuzzy system, $y_{d}$ is the optimal output and $K$ is the total number of data.

\section{Control scheme}

The general control design of the converter is shown in Figure 2. In the control method, the error signal which is generated from difference of output voltage and reference voltage, feds into the classic/fuzzy controller. The output of controller goes to the well-known Pulse Width Modulator (PWM) to determine the situation of switches, ${ }^{S_{1}}$ and $S_{2}$.

As noted in Section (2.2), the non-inverting buck-boost converter displays inherently different behavior in its two distinct operation modes.

The converter is completely stable in buck mode. Thus, the desired response speed can be gained by selecting wide bandwidth for the controller. However, due to the presence of the Right Half Plan zero and inappropriate stability margins in boost mode, the controller has to design with narrow bandwidth that slows the dynamic of the controller.

According to the above description, the worst-condition of the system, which occurs in boost mode, should be considered in designing a single controller for different operation modes of the converter.Among the classic controllers, PI controller has been used in indutrial and real-world application.

The classic PI controller is optimally designed based on the well-known frequency response method. The classic PI controller's formula is as follows [21]:

$$
V_{c t r l}(t)=K_{p} e(t)+K_{i} \int_{0}^{T} e(\tau) d \tau
$$

whereVctrl (t) is the controller output, $e(t)$ is the error signal, $K_{p}$ and $K_{i}$ are the proportional and integral gains, respectively. A schematic model of a classic PI controller is shown in Figure 3. 


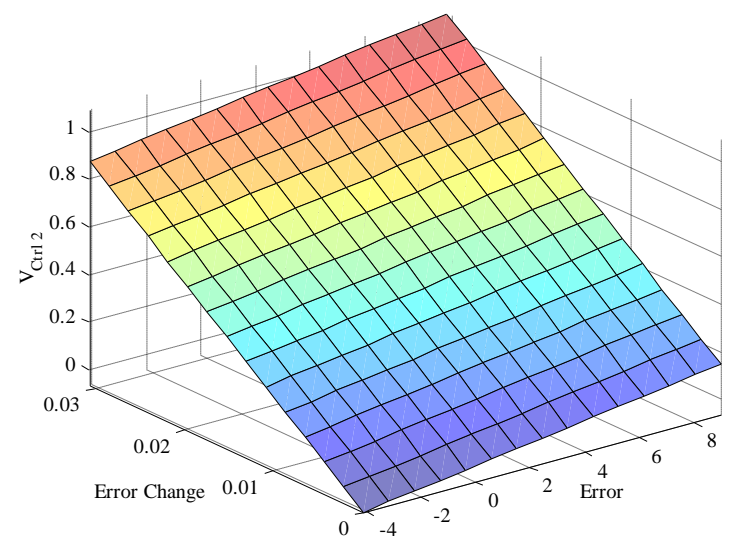

Figure 5- The output surface of the fuzzy PI controllers
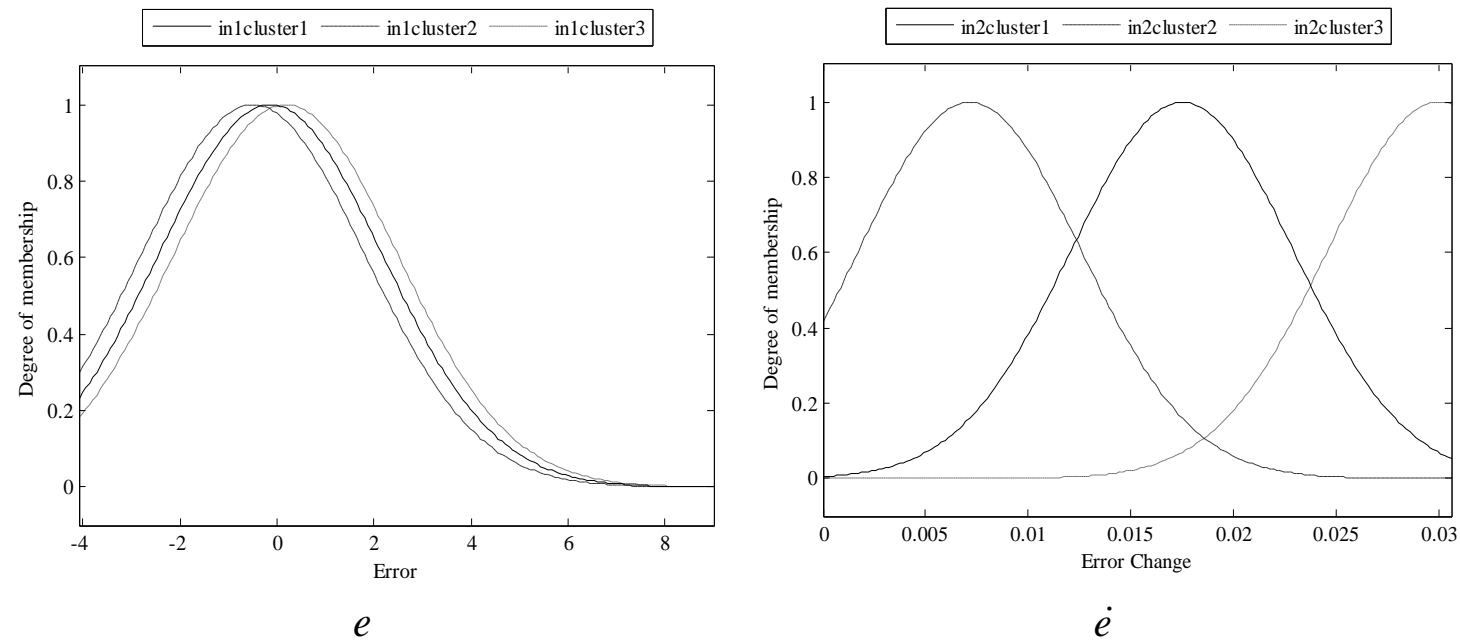

Figure 6- The membership functions of fuzzy PI controllers

Figure 6 shows the membership functions of fuzzy PI controllers. The fuzzy control rules in the form of (6) for fuzzy PI are as follows:

Rule 1)IFe is in1cluster1 and $\dot{e}$ is in2cluster1 THEN $y_{1}=0.01637 e+30.86 \dot{e}+4.125 e-6$

Rule 2)IFe is in1cluster2 and $\dot{e}$ is in2cluster2 THEN $y_{2}=0.01637 e+30.86 \dot{e}+4.57 e-6$

Rule 3)IFe is in1cluster3 and $\dot{e}$ is in2cluster3 THEN $y_{3}=0.01637 e+30.86 \dot{e}+4.10 e-6$

TSK fuzzy PI controller is designed using the input-output pairs of the classic PI controllers based on the method proposed in Section 3. Figure 4 shows the scheme of the proposed fuzzy PI controller. The relation between inputs and outputs of fuzzy $\mathrm{PI}$ is presented in Figure 5.

Table 2-Parameters and characteristics of time-response for classic PI and fuzzy PI controllers

\begin{tabular}{lll}
\hline Output Parameters & Classic & Fuzzy \\
\hline $\mathbf{K}_{\mathbf{p}}$ & 0.0163 & 1.5 \\
$\mathbf{K}_{\mathbf{i}}$ & 29.8603 & 1.6 \\
$\mathbf{t}_{\mathbf{r}}(\mathbf{s e c})$ & 0.0092 & 0.0051 \\
$\mathbf{t}_{\mathbf{s}}(\mathbf{s e c})$ & 0.0169 & 0.0099 \\
O.S. (\%) & 0.3463 & 0.3465 \\
U.S. (\%) & 0 & 0 \\
\hline
\end{tabular}


Table 3-Simulation parameters of non-inverting buck-boost Converter

\begin{tabular}{ll}
\hline $\mathbf{V}_{\text {in }}$ & $10 \mathrm{~V}$ \\
$\mathbf{V}_{\mathbf{o}}$ & $8,15,6 \mathrm{~V}$ \\
$\mathbf{f}_{\mathbf{s}}$ & $5 \mathrm{KHz}$ \\
$\mathbf{L}$ & $50 \mu \mathrm{H}$ \\
$\mathbf{R}_{\mathbf{L}}$ & $120 \mathrm{~m} \Omega$ \\
$\mathbf{R}_{\mathbf{o}}$ & $2 \Omega$ \\
$\mathbf{C}$ & $1.8 \mathrm{mf}$ \\
$\mathbf{R}_{\mathbf{C}}$ & $5 \mathrm{~m} \Omega$ \\
$\mathbf{R}_{\mathbf{S 1}}=\mathbf{R}_{\mathbf{S 2}}$ & $7.8 \mathrm{~m} \Omega$ \\
$\mathbf{R}_{\mathbf{D} 1}=\mathbf{R}_{\mathbf{D} 2}$ & $40 \mathrm{~m} \Omega$ \\
\hline
\end{tabular}

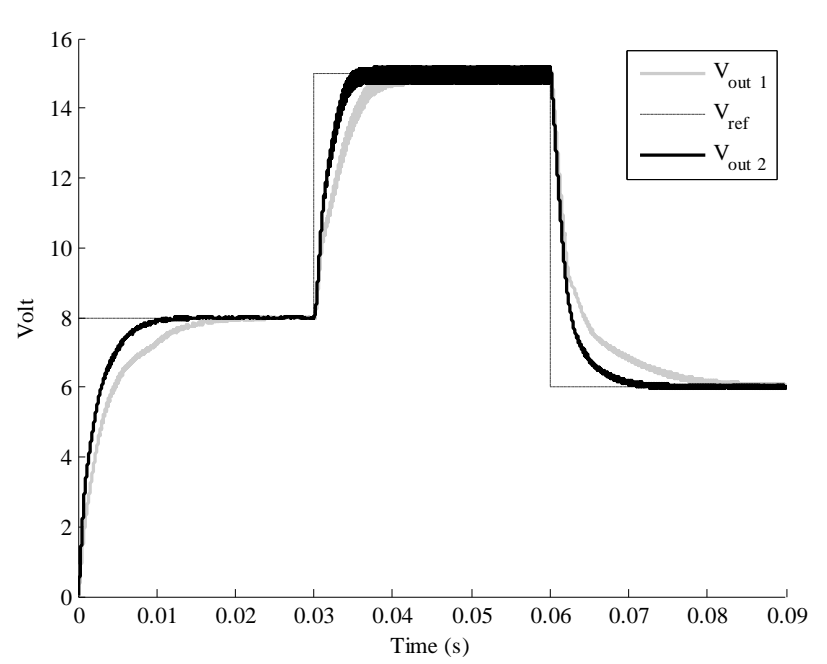

Figure 7-Comparing classic and fuzzy PI controllers

\section{Simulation and experimental results}

In order to evaluate the performance of classic and fuzzy PI controllers, they are compared with each other. Table 2 shows the time-response characteristic parameters of class and fuzzy controller. In Figure 7, the classic and fuzzy controllers are compared in controlling the converter. Based on the figure, the fuzzy $\mathrm{PI}$ in both buck and boost mode of converter has better performance in comparison with classic PI controller. The fuzzy PI controller has lower raising-time and settling-time.

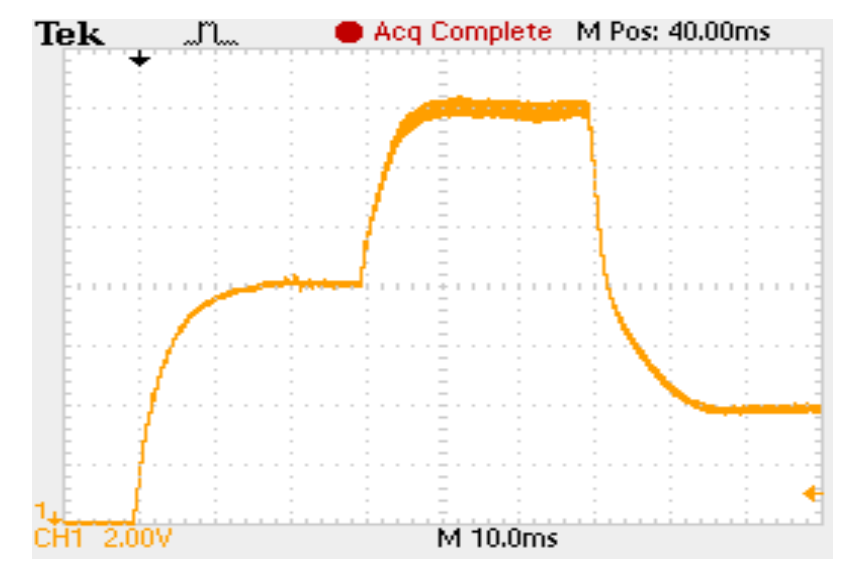

Figure 8- Experimental waveforms of output voltage using the classic controller 


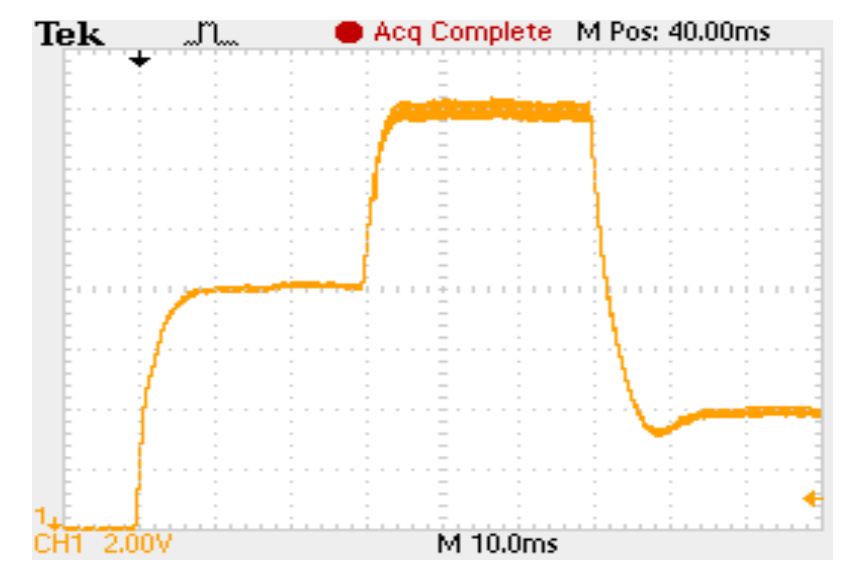

Figure 9- Experimental waveforms of output voltage using fuzzy controller

The values of converter's parameters using in the simulations and the experimental test are shown in Table3 where $R_{S}$ and $R_{D}$ are switches and diodes resistances respectively.

Figure 8 and Figure 9provide the experimental waveforms when the reference voltage changes from 8 to $14 \mathrm{~V}$ and then decreased to $4 \mathrm{~V}$ for classic and fuzzy controller, respectively.

\section{Conclusion}

In this paper, first the different operation modes of the non-inverting buck-boost converter were studied. Then, the linearized averaged state-space model for different operation modes was presented and stability margin of these models was investigated. An optimal T-S fuzzy PI controller for controlling the output voltage of the non-inverting buck-boost converter under variable reference voltageswas proposed. The subtractive clustering method was used to extract the initial T$S$ fuzzy system based on both conscious and subconscious knowledge of input-out pairs data, then RLS was used to regulate the coefficients of the consequent part of fuzzy rules.Both simulation and practical experiment results have verified that the proposed fuzzy PI controller is effective for the non-inverting buck-boost converter.

\section{ACKNOWLEDGEMENTS}

The authors would like thank the Power Electronic Laboratory in the Department of Electrical Engineering, FerdowsiUniversity of Mashhad, Iran, where the experimental part of this paper has carried out.

\section{References}

[1] B. Sahu,G.A. Rincon-Mora, A Low Voltage, Dynamic, Non-inverting, Synchronous Buck-Boost Converter for Portable Applications, IEEE Trans. Power Electron. 19 (2004) 443-452.

[2] H. Xiao, S. Xie, Interleaving double-switch buck-boost converter, IET Power Electron.(2012) 5899908. 
[3] R.F. Coelho, F.M. Concer, D.C. Martins, Analytical and Experimental Analysis of DC-DC Converters in Photovoltaic Maximum Power Point Tracking Applications, In IECON 2010-36th Annual Conference on IEEE Industrial Electronics Society. (2010) 2778-2783.

[4] V. Fereshtehpoor, Improvement in Power Factor Correction Capability in the Single Phase Noninverting Buck-Boost Converter, Master's science thesis, Dept. Electrical Eng., Science and Research Branch of Islamic Azad University, Tehran, 2012.

[5] Y. Lee, A. Khaligh, A. Emadi, A Compensation Technique for Smooth Transitions in a Non-inverting Buck-Boost Converter, IEEE Trans. Power Electron. 24 (2009) 1002-1015.

[6] E. Schaltz, P.O. Rasmussen, A. Khaligh, Non-Inverting buck-boost converter for fuel cell applications, In Industrial Electronics, 2008. IECON 2008. 34th Annual Conference of IEEE.(2008) 855-860.

[7] N. Mohan, T.M. Undeland, W.P. Robbins, Power Electronics Converters, Applications, and Design, J. Wiley, 3rd ed., 2003.

[8] R.D. Middlebrook, S.R. Cuk, A general unified approach to modeling switching converter power stages, In Power Electronics Specialists Conference. 1 (1976) 18-34.

[9] T. Takagi, M. Sugeno, Fuzzy identification of systems and its applications to modeling and control,IEEE Trans. Systems Man Cybernet. 15 (1985) 116-132.

[10] W.L. Xin, A Course in Fuzzy Systems and Control. Englewood Cliffs, NJ: Prentice-Hall, 1996.

[11] S. Guillaume, Designing fuzzy inference systems from data: An interpretability-oriented review. IEEE Trans. Fuzzy Sys. 9 (2001) 426-443.

[12] C. Hung, L. Huang, Extracting Rules from Optimal Clusters of Self-Organizing Maps, Int.Conf. on Computer Modeling and Simulation.1 (2010) 382-386.

[13] M. Setnes, Supervised fuzzy clustering for rule extraction, IEEE Trans. fuzzy sys. 8 (2000) 416-424.

[14] A.Priyono, M. Ridwan, A.J. Alias, R.A. OK Rahmat, A. Hassan, M.A. Mohd Ali, Generation of fuzzy rules with subtractive clustering, Journal Technology. 43 (2012) 143-153.

[15] C. Restrepo, J. Calvente, A. Cid-Pastor, A. E. Aroudi, R. Giral, A Noninverting Buck-Boost DC-DC Switching Converter With High Efficiency and Wide Bandwidth, IEEE Trans. Power Electron.26 (2011) 2490-2503.

[16] H.K. Lam, S.-C Tan, Stability analysis of fuzzy-model-based control systems: application on regulation of switching DC-DC converter, IET Control Theory \& Applications. 3 (2009) 1093-1106.

[17] R. Erickson, D. Maksimovic, Fundamental of power electronics, Springer, 2nd ed. 2000.

[18] J.J. Buckley, Sugeno type controllers are universal controllers,Fuzzy Sets and Systems. 53 (1993) 299-303.

[19] R. Qi, M. A. Brdys, Stable indirect adaptive control based on discrete-time T-S fuzzy model, FuzzySets and Systems. 159 (2008) 900-925.

[20] S. Chiu, Extracting fuzzy rules from data for function approximation and pattern classification, Fuzzy Information Engineering: A Guided Tour of Application. D. Dubois, H. Prade and R. Yager(eds), John Wiley Sons, 1997.

[21] K. Ogata, Modern control engineering, Prentice Hall, 5th ed., 2010. 